\title{
Hiperconectividad y Psicoanálisis
}

\section{Hyperconnectivity and Psychoanalysis}

\author{
DE LA MORA-ESPINOSA, Rosa Imelda*† \& ORTEGA-MARTÍNEZ, María Cristina \\ Facultad de Psicología de la Universidad Autónoma de Querétaro (UAQ). Centro Universitario, Cerro de las Campanas \\ S/N, 76010, Santiago de Querétaro, Querétaro
}

ID 1er Autor: Rosa Imelda, De La Mora-Espinosa / ORC ID: 0000-0003-4010-2223, Researcher ID Thomson: S-49222018, CVU CONACYT ID: 389065

ID 1er Coautor: María Cristina, Ortega-Martínez / ORC ID: 0000-0002-6188-4677, CVU CONACYT ID: 597320

DOI: $10.35429 / J T M S .2019 .17 .5 .23 .33$

Recibido 11 de Agosto 2019; Aceptado 30 Diciembre, 2019

\begin{abstract}
Resumen
Objetivos: Abordar problemas y perspectivas del psicoanálisis en la actualidad, en esta era hipermoderna de hiperconectividad y nuevas tecnologías, dado que en la clínica psicoanalítica se presentan hoy síntomas nuevos por el impacto de la hiperconectividad y es menester que el psicoanálisis siga dando soporte a los sufrimientos psíquicos de los sujetos humanos. Metodología: Realizar un entrecruce de los escritos nuevos de psicoanálisis y subjetividad en esta era hipermoderna e hiperconectada, recuperando los fundamentos del psicoanálisis que fueron establecidos por Freud, a saber, el inconsciente, la transferencia, la demanda y la asociación libre y posteriormente trabajados por Lacan, quien también abordó las complicaciones que surgen en la subjetividad por el uso de gadgets, complejidades advertidas por Lacan en La tercera (1974). Contribución: Puntualizar perspectivas y problemas del psicoanálisis en la actualidad en esta era hipermoderna y de hiperconectividad, para pensar la clínica psicoanalítica con estos nuevos síntomas y modalidades, dado que hoy se nos invita a gozar en muchas formas y el psicoanálisis nos posibilita no sentirnos culpables por no gozar, en virtud de que en la vida actual hay un deber ético de gozar (Zizec 2006/2013).
\end{abstract}

Gozar, Hiperconectividad, Psicoanálisis

\begin{abstract}
Objectives: Tackling problems and prospects of psychoanalysis today, this was hypermodern hyperconnectivity and new technologies, since in the psychoanalytical clinic by the impact of the hypernonnectivity new symptoms occur today and it is need that psychoanalysis continue to give support to the mental suffering of human subjects. Methodology: Make a crossing of the new writings of psychoanalysis and subjectivity in this era, hyperconnected and hypermodern recovering the foundations of psychoanalysis which were established by Freud, namely, the unconscious, the transfer, the free association and demand and later worked on by Lacan, who also addressed the complications that arise in the subjectivity by the use of gadgets, complexities instructed by Lacan in The Third (1974). Contribution: Point out prospects and problems of psychoanalysis currently in this hypermodern era and hyperconnectivity, to think the psychoanalytical clinic with new symptoms and modalities, since today we are invited to enjoy in many ways and the Psychoanalysis enables us to not feel guilty for not enjoy, by virtue that in actual life there is an ethical duty to enjoy (Zizec $2006 / 2013)$.
\end{abstract}

Enjoy, Hyperconnectivity, Psychoanalysis

Citación: DE LA MORA-ESPINOSA, Rosa Imelda \& ORTEGA-MARTÍNEZ, María Cristina. Hiperconectividad y Psicoanálisis. Revista Transdisciplinaria de Estudios Migratorios. 2019, 5-17: 23-33

\footnotetext{
* Correspondencia al Autor (correo electrónico: rosidelamora@yahoo.com.mx)

$\dagger$ Investigador contribuyendo como primer autor.
} 


\section{Introducción}

Vivimos una época que está permeada por las nuevas tecnologías y los fenómenos que se derivan de ellas. Estamos en una era que se ha dado en llamar hipermodernidad en virtud del desarrollo vertiginoso, constante y continuo de tecnologías, una de las características de esta época es lo que se le ha dado el nombre de hiperconectividad.

Para darnos una idea de los alcances de la hiperconectividad a través del internet en el World Wide Web, se calcula que, en la actualidad de los más de 7 mil millones de habitantes de la Tierra, hay alrededor de 4 mil millones de usuarios de internet, es decir más de la mitad de la población, 57,14\% (García Sánchez, 2019), lo cual nos da la idea de que muchísimos seres humanos alrededor del planeta están involucrados de una manera o de otra en el internet. Situación que no prevalece en la Deep $W e b$, en ella se calcula que hay alrededor de dos millones de usuarios, (García Sánchez, 2019), una cifra bastante menor que la de los internautas que están en la "superficie" de internet.

En nuestro país el Instituo Nacional de Estadística Geografía e Informática INEGI nos devela que somos 125 millones de mexicanos (Granados, 2019), de los cuáles, se calcula que 82.7 millones de mexicanos son usuarios de internet, (Riquelme, 2019), lo que representa el $66.16 \%$ de la población mexicana. Se estima que los internautas mexicanos están conectados a la red un tiempo promedio de 8 horas y 20 minutos al día, mucho más del tiempo que ven televisión, que se calcula en 2 horas y 20 minutos y el radio 2 horas. Los principales usos de internet de los mexicanos, utlizando primordialmente teléfonos inteligentes, son redes sociales y servicios de mensajería instantánea, y en segundo término usos de banca y servicios financieros en línea

La hiperconectividad conlleva una serie de fenómenos insoslayables e inéditos, a saber, con una tablet, y/o con un teléfono inteligente (smart phone) y más si está enlazado a un reloj inteligente (smart watch) que prácticamente funcionan como una mini computadora en la palma de la mano y/o en la muñeca, si contamos con el servicio de internet y/o nos conectamos a algún servidor gratuito de internet, podemos tener acceso a billones de páginas de internet con inconmensurables conocimientos de múltiples ciencias y disciplinas.
Así como a nuestras cuentas de mensajería instantánea, de correos, electrónicos, nuestras cuentas de redes sociales, cuentas de banca electrónica, acceso a las últimas noticias, además de que podemos tomar y transmitir en tiempo real fotografías, videos y grabaciones de manera inmediata. Inconmensurables trámites escolares, laborales, de viajes, de documentos de vida, entre otros, se realizan a través del internet.

Toda la actividad de los internautas se registra en la nube, aunado al hecho de que sabemos que en el internet no hay nada privado, y que los gobiernos espían a los internautas; como nos lo han hecho saber Julian Assange, creador de Wikileaks (actualmente encarcelado en la prisión de Belmarsh en Londres, Reino Unido), Edward Snowden, (antiguo empleado de la Central Intelligence Agency conocida por sus siglas CIA y de la National Security Agency cuyas siglas son NSA, actualmente asilado en Rusia) y Martin Hilbert. (profesor adjunto del Departamento de Comunicación de la Universidad de California, EEUU.)

Aunado a lo anterior, en el internet opera lo que se conoce como Inteligencia artificial, (conocida por sus siglas en inglés como IA), son programas capaces de ser ejecutados independientemente de la máquina $\mathrm{o}$ computadora que lo ejecute, de este modo la IA "elige" los contenidos que supuestamente nos interesan porque "lee" diversas páginas en internet en las que navegamos. Sin embargo, estos programas no nos permitirían ver otros contenidos que a lo mejor nos interesan, pero no aparecen porque la IA no los "eligió". Es así como funcionan Google, Siri, Apple, Facebook, Amazon, Twitter, YouTube; si bien estas compañías dan a conocer sus políticas de privacidad, también se reservan el derecho de manejar la información que tienen de todos sus usuarios.

Esta situación infortunadamente conlleva complicaciones, la más reciente involucra a Google, compañía que será multada por la Comisión Federal de Comercio de EEUU (FTC por sus siglas en inglés) por la cantidad de 170 millones dólares por violar la privacidad de los niños en YouTube, en virtud de que "recopilaba datos personales de menores de edad sin consentimiento de sus padres." (Lermann y Gordon, 2019). 
Esa misma Comisión ya había multado a Facebook con 5000 millones de dólares por violación de la privacidad de sus usarios. Ya es bastante grave que se recopilen datos de los internautas, pero recopilar datos de niños es más que escandaloso. Aparte de la multa que les aplican a esas compañías, surgen diversas preguntas, ¿qué hacen con esa información?, ¿para qué la van a utilizar en el futuro?, ¿a quién o quienes se la pueden proporcionar?, posteriormente a la multa, ¿eliminarán toda la información que poseen para evitar contrariedades futuras?

Como toda la actividad en internet se registra en la "nube", esta no significa que los contenidos virtuales estén en "el cielo", la nube implica una tecnología de servidores y computadoras:

La nube se entiende conceptualmente como un intercambio digital de bits, pero en realidad se trata de infraestructura física: centros de datos del tamaño de un hangar y cables transoceánicos que transportan petabytes de información. Una costosa infraestructura arrendada a las empresas para satisfacer sus necesidades informáticas, para que Netflix transmita películas a tu teléfono sin problemas o Citibank procese miles de millones de transacciones en línea" (Carr y Bass, 2019, p. 21)

Es por ello que las compañías que ofrecen servicios de internet se han vuelto sumamente rentables. Estas compañías en México, ofrecen, en los últimos tiempos, distintos paquetes de servicios de internet que van desde 10 megas hasta 200 megas, con el correspondiente aumento de las cuotas, mientras más megas, mayor es el costo. En contraste, hace varios años los habitantes de Canadá ya contaban con servicios de internet de más de 100 megas. En Europa los servicios de internet son de calidad, por ejemplo, en Alemania un hotel de 4 estrellas ${ }^{1}$, ofrece un servicio de internet de 300 megabytes a sus huéspedes, ... sic
Esta hiperconectividad nos conduce a situaciones complejas en virtud de que, si bien tenemos la bondad de saber en tiempo real los mensajes, noticias, correos electrónicos, notificaciones bancarias que nos están llegando, simultáneamente es complicado atender un sinnúmero de notificaciones de lo más diverso, y en ocasiones es difícil atender de manera inmediata las notificaciones y responder de prisa, en virtud de que nuestras actividades cotidianas, como trabajar, estudiar, comer, asearnos, transportarnos no nos permiten responder con la inmediatez que a veces se nos requiere. Aunque es innegable que hay internautas que están conectados y activos con todo y las actividades cotidianas.

Es insoslayable que la hiperconectividad tiene muchas ventajas, veamos algunas de ellas, las instituciones bancarias nos avisan en tiempo real los movimientos ya sea de retiro y/o depósito que se están llevando a cabo; si uno accede a un servicio de transporte existen aplicaciones con las que uno puede enviarles a sus contactos de confianza la ruta que está siguiendo el automóvil, sobre todo para evitar alguna acción delictuosa como ser secuestrado. También hay aplicaciones para saber en dónde están, en tiempo real, nuestros hijos, nuestros padres, nuestros familiares y amigos. Incluso En la última semana del mes de agosto de 2019 Facebook creó una aplicación de alerta para tiroteos y fenómenos naturales. (Noticias Telemundo, 2019)

De lo más reciente que se ha producido, podemos comentar que alumnos del Centro de Estudios Científicos y Tecnológicos (CECyT) No. 13 "Ricardo Flores Magón" del Instituto Politécnico Nacional, de Ciudad de México crearon un software para enfermos de Alzheimer, se llama Heizmer, y “... estimula las funciones cognitivas, al mismo tiempo registra el avance del padecimiento y permite la ubicación del enfermo en tiempo real para que sus familiares puedan saber con exactitud dónde se encuentra." Asimismo,

Este programa informático aporta un mejor control sobre el avance de la enfermedad, porque incluye dinámicas personalizadas basadas en la información del paciente como: nombres de familiares, actividades, experiencias y lugares significativos para la persona con Alzheimer. (Sin Embargo, 12 julio 2019).

\footnotetext{
${ }^{1}$ Hotel Intercity Berlin Haupbahnhof
} 
Este es un software realmente importante en virtud de que se calcula que hay alrededor de 800,000 mexicanos con Alzheimer, evidentemente los pacientes que se verían beneficiados son aquellos que cuentan con smarphones. Y podríamos mencionar un sinnúmero más de programas de software y aplicaciones que nos brindan beneficios y nos mantienen hiperconectados allende las fronteras.

\section{Algunas complicaciones de la hiperconectividad}

"Technology is neither good or bad; nor is it neutral",

Melvin Kranzberg, 1986

Historiador estadounidense

(La tecnología no es ni buena, ni mala, pero tampoco es neutral)

$\mathrm{Si}$ bien estar inmersos en esta hiperconectividad nos permite "estar al día" de todo lo que está aconteciendo en nuestro planeta, y en nuestras cuentas bancarias, de correo, de redes sociales, no obstante, recibir innumerables notificaciones puede producirnos diversas complicaciones subjetivas, en primera instancia, uno podría angustiarse ante la imposibilidad de responder a mensajes en virtud de que las actividades laborales, escolares y cotidianas no nos lo permiten. Otra situación es que si llegan muchísimos mensajes (por ejemplo, de WhatsApp), no hay posibilidades de leerlos todos, y en ocasiones uno podría no revisar mensajes realmente importantes.

Evidentemente en el internet contamos con una gran cantidad de información de los más diversos temas, sin embargo, una complicación que se presenta es cuando uno teclea un tema que a uno le interesa en un navegador, y aparecen millones de páginas web relacionadas al tema y entonces es preciso navegar mucho tiempo para poder discriminar la información que realmente sea relevante. Otra complicación insoslayable de la hiperconectividad consiste en las fake news, que muchas veces circulan y se vuelven virales e infortunadamente muchos internautas las replican sin mayor reflexión.
Como muestra de una fake news que tuvo consecuencias gravísimas, fue un artículo que el ex-médico inglés Andrew Jeremy Wakefield escribió y publicó en internet en 1998 diciendo que la vacuna triple vírica contra sarampión, paperas y rubeola estaba relacionada con el autismo. La noticia se hizo viral sobre todo a través del internet, el resultado fue catastrófico, muchos padres de familia no vacunaron a sus hijos, y se presentaron enfermedades que a finales del siglo XX ya se consideraban extintas, como el sarampión, que en la actualidad sigue cobrando víctimas. Ulteriormente se demostró que lo que afirmaba Wakefield no era cierto y le revocaron su licencia de médico en 2010.

Asimismo, no podemos olvidar que existen stalkers en el internet que se dedican al ciberbulling, de ahí que cuando un sujeto humano es acosado en el internet, las consecuencias son catastróficas e inconmensurables, en virtud de que en el internet no hay nada privado y todo permanece, de ahí que una vez que se ha publicado un post altisonante sobre alguien, eso perdura hasta el fin de los tiempos mientras continuemos con el internet.

Nos encontramos en un ambiente contrario a la intimidad, ahora se habla de extimidad, concepto creado por Lacan, para explicitar como lo más íntimo del sujeto humano es a la vez lo más exterior. Este término de extimidad, posteriormente es retomado por Sergé Tisserón, (2001) psiquiatra y psicoanalista francés, para nominar la sobre exposición de la intimidad en la que se ven inmersos muchos de los internautas, escenario que puede llegar a ser enloquecedor para más de alguno.

$\mathrm{Si}$ bien nos podemos enterar de inconmensurables conocimientos, noticas, entre otras cosas, y podemos estar "al día", tampoco podemos olvidar que no todas las notas en internet son buenas noticias, que uno puede enterase de noticias muy malas que nos pueden producir angustia y sufrimiento.

Asimismo, el internet permite enterarse de manera ultrarrápida de en dónde va a realizarse una reunión para divertirse, también para aquellos que hacen resistencia social frente a los embates de sus gobiernos, les permite comunicarse rápidamente y saber qué hacer. 
Esta era de la hipermodernidad e hiperconectividad está conduciendo a los sujetos humanos a escenarios antes insospechados de goces irrestrictos y simultáneamente a no saber qué hacer al respecto, en virtud de que los seres humanos no podemos olvidar que somos mortales y sexuados. De este modo, cuando estamos navegando podemos tener una sensación de completud, de placer inmenso, de narcisismo extremo, de momentos lúdicos, sin embargo, únicamente estamos "viviendo el mundo en una pantalla", sin que medie de por medio otro sujeto humano con el cual tener una interlocución más acorde a lo que es realmente la vida humana.

$\mathrm{Al}$ respecto recuperamos las seis leyes de la tecnología del historiador estadounidense Melvin Kranzberg, (1986) a saber:

1. La tecnología no es ni buena ni mala, pero tampoco es neutral.

2. La invención es la madre de la necesidad.

3. La tecnología viene en paquetes, grandes y pequeños.

4. Aunque la tecnología puede ser un elemento principal en muchos asuntos públicos, factores no tecnológicos tienen prioridad en las decisiones de política tecnológica.

5. Toda la historia es relevante, pero la historia de la tecnología es la más relevante.

6. La tecnología es una actividad muy humana - y así es la historia de la tecnología.

Estas leyes de Kranzberg son insoslayables en esta investigación en virtud de la enorme importancia que tiene la tecnología, en su estrecha relación con la subjetividad humana, relación que es más intensa en el presente, y es justamente la subjetividad humana cuando es trastocada por padecimientos psíquicos, la que es abordada y soportada por el psicoanálisis.

Es innegable que realmente precisamos de otros seres humanos para proseguir en esta aventura que llamamos vida, y cuando nuestros deseos y padecimientos se vuelven difíciles, complicados, nos producen angustia, es precisamente en el psicoanálisis en dónde tienen cabida, ya que, en este, se atiende a los sujetos humanos cuando tienen padecimientos subjetivos.
El psicoanálisis brinda un espacio de soporte y atención de ese sufrimiento psíquico. En palabras de Zizek:

El goce funciona hoy como un extraño deber ético: los individuos no se sienten culpables por violar alguna prohibición moral practicando placeres ilícitos, sino por no ser capaces de gozar. En esta situación, el psicoanálisis es el único discurso que autoriza a no gozar -no proscribe el goce, sólo nos alivia de la presión de tener que cumplir con él. (Zizek, 2006/2013, p. 111)

Indudablemente el goce al que se refiere Zizek conlleva al gozar de grandes placeres que nos brinda la vida, placeres de los que dice este filósofo que no podemos dejar de sentirnos constreñidos a ejercerlos, de no ser así, podemos sentirnos culpables.

Esta manera de goce dilucidado por Zizek es diferente al goce que habla Lacan, en la enseñanza de este psicoanalista francés el principio del placer es el que pone límite al goce, simultáneamente el sujeto trata de trasgredir las prohibiciones impuestas a su goce e ir más allá del principio del placer, pero al traspasar este principio lo que el sujeto encuentra es dolor, porque el sujeto sólo puede soportar cierta cantidad de placer, después de ello el placer se torna en dolor, ese placer doloroso es el goce, el goce es sufrimiento, es la satisfacción que obtiene el sujeto de su síntoma.

Es justamente en su Conferencia de 1966 Psicoanálisis y Medicina que Lacan puntualiza que: "un cuerpo es algo que está hecho para gozar, gozar de sí mismo" (Lacan, 1966) y esto es innegable, diariamente sentimos en el cuerpo, no necesariamente gozando, luego también sentimos dolor, sufrimiento, penas, y sí, se siente en el cuerpo.

En la misma conferencia Lacan hace una distinción muy precisa de la diferencia entre placer y goce, nos dice: 
¿Qué se nos dice del placer? - que es la menor excitación, lo que hace desaparecer la tensión, lo que más la atempera, es decir, lo que nos detiene necesariamente en un punto de lejanía, a muy respetuosa distancia del goce. Pues lo que yo llamo goce en el sentido en que el cuerpo se experimenta, siempre es del orden de la tensión, del forzamiento, del gasto, incluso de la hazaña. Indiscutiblemente hay goce en el nivel en que comienza a aparecer el dolor, y sabemos que es solamente a ese nivel del dolor que puede experimentarse toda una dimensión del organismo que de otro modo permanece velada. (Lacan, 1966)

En este tenor, vamos elucidando algunos de los placeres de estar hiperconectados y a la vez el goce del que nos habla Lacan que también puede aparecer con esta hiperconección de los sujetos, debido a que nuestras vidas cotidianas están siendo trastocadas por estas nuevas tecnologías, con las que por momentos tenemos una sensación ilusoria de completud, que es únicamente eso, una ilusión. No obstante, no podemos olvidar que somos mortales y sexuados; y que nos hacen falta otros sujetos humanos para transitar por este complejo camino que llamamos vida.

\section{Algunos síntomas nuevos en la era de la hiperconectividad}

Si bien en la actualidad estamos hiperconectados, simultánea e infortunadamente en algunos países, ha ido disminuyendo la vida en comunidad; incluso hoy la soledad se considera un mal de nuestro tiempo, ya sea por complicaciones familiares, muchos hijos ya no se hacen cargo de sus padres que son adultos mayores, que mientras tengan salud física no hay mucho problema, pero si se enferman o tienen algún accidente las cosas se complican.

También hay soledad por elección propia, pues hay seres humanos que prefieren vivir solos e incluso hay quienes hacen home office, domicilian todos sus pagos, y tienen una relación precaria con sus vecinos.

Pero la vida es solo la vida, nunca falta que hasta al más solitario se le invite a una reunión familiar y/o social, y entonces aparece un problema, ¿con quién ir?, ahí entran un nuevo tipo de negocios, a saber, en los Estados Unidos y en Japón se venden servicios de amigos y de abrazos (Laborde, 2019)
En nuestros países latinos todavía nos gusta, por usos y costumbres, reunirnos, tocarnos, abrazarnos y somos festivos, esperemos no llegar a esos niveles de soledad que son más frecuentes en los países del primer mundo.

Los seres humanos, como bien nos ha mostrado el psicoanálisis, necesitamos de otros para sobrevivir, para ser humanos, necesitamos que nos hablen, que nos acaricien, necesitamos compartir nuestras vivencias, nuestros pensamientos, nuestros sentimientos. Si ello no ocurre no es de extrañar que se presenten depresiones, entre otros problemas subjetivos.

En cuanto a la depresión, ella consiste en un padecimiento subjetivo, que ya se considera un problema de salud pública en los últimos tiempos, circunstancia que ha sido denunciada por la Organización Mundial de la Salud, se calcula que el $4 \%$ de la población mundial la padece, alrededor de 350 millones sufren de depresión, los más proclives son las mujeres, los jóvenes y los ancianos. Se caracteriza por: "la presencia de tristeza, pérdida de interés o placer, sentimientos de culpa o falta de autoestima, trastornos del sueño o del apetito, sensación de cansancio y falta de concentración. Este trastorno puede llegar a hacerse crónico o recurrente $\mathrm{y}$ en su forma más grave, puede conducir al suicidio." (Gobierno de México, 2016)

En el mundo, este trastorno representa la cuarta causa de discapacidad en cuanto a la pérdida de años de vida saludables. En México, ocupa el primer lugar de discapacidad para las mujeres y el noveno para los hombres. Además se estima que $9.2 \%$ de la población ha sufrido depresión, que una de cada cinco personas sufrirá depresión antes de los 75 años y que los jóvenes presentan tasas mayores. (Gobierno de México, 2016).

Un espacio que precisamente ayuda a las personas deprimidas es el psicoanálisis, ahí se les puede dar soporte a sus sentimientos de tristeza, a sus angustias. 
Otros padecimientos subjetivos que también son más frecuentes en la actualidad son la anorexia y la bulimia, para lo cual en el internet hay una serie de páginas dedicadas a estos trastornos en las que se ofrecen tips y acciones para seguir siendo bulímico $\mathrm{y} / \mathrm{o}$ anoréctico y que los familiares y amigos percaten de dicha situación, debido a que los familiares y amigos les produce angustia en virtud del peligro de perder la vida que conlleva sobre todo la anorexia. Al respecto pueden consultarse las páginas de Mía y Ana, Mía hace referencia a la bulimia y Ana a la anorexia. (Ana y Mía, 2019). Más recientemente Ana y Mía se han trasladado al sistema de mensajería instantánea WhatsApp, con la complicación de que familiares y amigos que no estén registrados en dichos grupos no podrían saber lo que está pasando.

Infortunadamente también en el internet hay páginas web que explicitan como suicidarse, más de alguno las han consultado para quitarse la vida, sabemos de casos de sujetos que después de la información obtenida en internet lo han cometido, dejando un gran dolor y tristeza a familiares, amigos y conocidos.

Además de lo anterior, en estos tiempos en que el internet brinda cantidades inconmensurables de información, se presenta de manera cada vez más frecuente que algunos sujetos buscan en el World Wide Web toda la información posible sobre sus sufrimientos subjetivos, así por ejemplo, si alguien padece de una fobia a las ratas, busca qué es, cómo se presenta, posibles soluciones al respecto. Incluso solicitar atención clínica virtual a pesar de los inconvenientes de extimidad.

Esto es tan sólo una pequeña muestra de padecimientos subjetivos actuales, para mostrar algunas de las complicaciones que se producen en el presente en la hipermodernidad.

\section{Del Psicoanálisis}

En otra escritura, hemos planteado que en la última década cada vez es más frecuente realizar psicoanálisis a distancia y/o de manera virtual online, (De La Mora y Rosales, 2013), ya sea por dificultades económicas, o por enfermedades, viajes o cambios de residencia, con las complicaciones correspondientes de la dificultad de mantener la secresía.
Incluso con los sistemas de internet del presente, una sesión psicoanalítica tradicional en consultorio, podría estarse escuchando y grabando a través de los teléfonos celulares, ya sea del paciente y/o del analista; sean smart phone o no, en tanto tengan micrófono y una señal radioteléfonica $o$ satelital se puede escuchar y grabar; debido a que los teléfonos celulares pueden ser intervenidos y transformados en sistemas para escuchar a sus dueños, grabarlos y saber su ubicación física. Apenas hace unos días, la compañía Apple admitió que su asistente virtual Siri, estuvo escuchando conversaciones sin autorización, al verse descubiertos, no tuvieron más alternativa que disculparse. (Infobae, 2019) A pesar de estos inconvenientes en la era de la hiperconectividad, en virtud de que en el psicoanálisis se trabaja en la intimidad y no se emiten prejuicios; en la clínica psicoanalítica existe la condición de posibilidad de hablar con el analista, que es un interlocutor experto, de todas las complicaciones subjetivas que nos aquejen, de todos nuestros "infiernos", de nuestras penas, de lo que no nos atrevemos a decir a nuestros mejores amigos.

Si queremos parafrasear un poco con el tema de esta investigación, en la sesión psicoanalítica podemos hablar de nuestras angustias por haber posteado una noticia que a nosotros nos parece importante y angustiarnos por no haber recibido "suficientes" likes, o de pasar conectados y en línea mucho tiempo sin atender otras actividades, o de estar de curiosos buscando información sobre nuestros congéneres, o de que nuestra intimidad sea sobreexpuesta en algún portal de internet que nos interesa que no haya información de nosotros, o de ver muchos posts en los que los internautas dicen que son felices, de suponer que debemos estar felices por las grandes comodidades que nos brinda el mundo actual, así como las facilidades que nos brinda la tecnología, y aun así encontrarnos como sujetos que realmente no logramos la completud a la que aspiramos, esa completud ilusoria que nos brindan los gadgets cuando estamos conectados en el mundo virtual, en una pantalla, encontrarnos con que somos seres en falta, incompletos, seres que podemos morir en cualquier momento, seres que precisamos amar y ser amados, seres que somos sexuados que asimismo precisamos una vida sexual lo más satisfactoria posible, ejercer nuestra erótica consensuada con otros. 
Reiteramos que, en el psicoanálisis, a través de la asociación libre, el analista puede atender la demanda del sujeto que sufre, hacerse cargo de las manifestaciones de su inconsciente y de su transferencia y que ambos pueden continuar el tratamiento psicoanalítico hasta que el sujeto pueda pasar a otra cosa, para que aquello que lo perturba ya no le cause más sufrimiento.

\section{Metodología a desarrollar}

Esta es una investigación básica teórica, trabajamos con libros y textos publicados, y el acceso a la información en el internet es del orden público. Los textos clásicos de psicoanálisis son públicos, así como las nuevas escrituras que se están produciendo sobre el psicoanálisis en la actualidad y sobre esta nueva era de la hiperconectividad, están publicados, en su mayoría en revistas digitalizadas y/o en páginas web de instituciones psicoanalíticas.

Implica realizar un cruce de escritos psicoanalíticos clásicos de Freud y Lacan, que nos hablan de fundamentos del psicoanálisis, a saber, el inconsciente, la transferencia, la demanda y la asociación libre; con nuevos escritos que se están produciendo sobre el psicoanálisis en la era de la hiperconectividad con las complicaciones que surgen en la subjetividad por el uso de gadgets, mismas que ya fueron advertidas por Lacan en La tercera $\left(1^{\circ}\right.$ de noviembre de 1974).

Esto involucra un abordaje metodológico de recolección, conceptualización, análisis e interpretación de los textos, contrastación, desde el marco teórico del psicoanálisis clásico; con escrituras nuevas de psicoanalistas que publican sobre la subjetividad a raíz del impacto que producen las nuevas tecnologías en esta era de hipermodernidad e hiperconectividad, así como de filósofos que abordan el psicoanálisis.

Se está construyendo una escritura que va perfilando las conclusiones del recorte de lo investigado, una escritura que está entrecruzando los problemas y perspectivas del psicoanálisis clásico; con el psicoanálisis en la actualidad en esta era hipermoderna y de hiperconectividad.

\section{Resultados}

Es importante seguir brindando a los sujetos la posibilidad de hablar de sí mismos, de sus sufrimientos, de sus avatares, de sus vicisitudes subjetivas que les producen sufrimiento psíquico, y de que estemos muy pendientes al respecto. En la actualidad es justamente el psicoanálisis uno de los espacios que brindan tal posibilidad.

Los resultados del psicoanálisis sólo pueden vislumbrarse como nos enseñó Freud "caso por caso", o como nos decía Lacan en su exquisita singularidad, y en cuanto un sujeto pueda orientarse hacia otros avatares en vez de estar complicado con sus sufrimientos psíquicos que no le permiten realizar sus actividades de manera más excelsa, es entonces que podemos hablar de resultados, aun a sabiendas de que puedan presentarse de nuevo situaciones complicadas y sufrientes en la vida, así, quien haya transitado por un análisis personal podrá encarar esas nuevas complejidades, si bien con sufrimiento, pero al mismo tiempo sabiendo que se puede salir avante.

\section{Agradecimiento}

En virtud de que esta investigación es de una problemática actual, agradecemos a la Universidad Autónoma de Querétaro, especialmente a la Facultad de Psicología, por darnos la oportunidad de realizar nuestras labores de investigación.

\section{Consideraciones finales}

Debido a que esta nueva era de hipermodernidad, de hiperconectividad trastoca nuestras vidas de una manera inédita, ultrarrápida, se presentan complicaciones propias de estas nuevas circunstancias.

Las posibilidades de elegir contenidos a libre demanda en el internet conllevan que el sujeto difícilmente se cuestione al respecto, sobre todo si no interacciona con otros, debido a que en cualquier momento puede desconectarse de una página web que le resulte discordante y volcarse a otra que sea más de su agrado. Y este "vivir el mundo en una pantalla", le puede brindar una alegría pasajera y narcisística como recibir muchos likes por un post que ha publicado, y/o encontrar una información que coincide con su manera de pensar. 
Sin embargo, esto es pasajero y finalmente no lo exime de sentir angustia, como tampoco lo exime de saberse un ser mortal y sexuado.

Estas nuevas tecnologías nos producen sensaciones diversas, como que cuando estamos inmersos en las pantallas de nuestros artefactos inteligentes nos dan la sensación de goce irrestricto y de completud, no obstante, no podemos olvidar que los sujetos humanos seguimos padeciendo sufrimientos psíquicos, de los cuáles puede hacerse cargo el psicoanálisis.

En esta era hipermoderna en la que estamos hiperconectados, aparecen nuevas complejidades en los sujetos y su forma de vivir cotidianamente, por ejemplo, ya en 1990, Lipovetsky nos comenta el gran problema del hiperindividualismo narcisista en el que el sujeto de hoy vive inmerso, en un hedonismo sin precedentes, con muchas libertades. Hedonismo que se ha exacerbado con la involucración de los sujetos humanos en el internet. Es una época en la que el mercado es el que impone la ley; una época hipermoderna en la que no hay oposición, normativa, regulación y aparte es global, y podemos avanzar a que justamente en el World Wide Web las posibilidades de buscar placeres son inconmensurables.

Parte de la problemática en la actualidad radica en que hoy hay un asunto de gozar, y justo el psicoanálisis como nos revela Zizek (2006/2013) nos alivia de la presión de no tener que cumplir con el goce, en virtud de que sigue siendo un espacio privado en el que el sujeto puede explayar sus deseos, demandas, sufrimientos psíquicos con un interlocutor adecuado, el analista, quien no lo juzga y le da sostenimiento.

De este modo, como seres humanos, en tanto en cualquier momento puede presentarse la condición de posibilidad de tener padecimientos subjetivos, precisamos de tener un espacio de intimidad con un interlocutor experto para trabajar sufrimientos de nuestra subjetividad, y uno de esos espacios en la actualidad, lo brinda el psicoanálisis.

\section{Referencias}

Allouch, J. (1992). 213 Ocurrencias con Jacques Lacan, (R. Trejo y P. Hernández Trad.) México: Ed. SITESA.
Ana y Mía, (2019) disponible en: https://ana-ymia-para-princesas7.webnode.es. 28 de junio de 2019, consultado el 2 de septiembre de 2019.

Bauman, Z. (2003/2017). Amor líquido. Acerca de la fragilidad de los vínculos humanos. (M. Rosenberg, J. Arrambide Trad.) México: FCE

Berthelsen, D. (1995) La vida cotidiana de Sigmund Freud y su familia. Recuerdos de Paula Fichtl. (P. Estelrich Trad.) Barcelona: Ediciones Península.

Carlino, R. (2010). Psicoanálisis a distancia, Buenos Aires: Ed. Lumen.

Carr A. y D. Bass. (2019). Satya Nadella lleva a Microsoft al renacimiento. Bloomberg Businessweek. El Financiero, 30 de mayo de 2019

De La Mora, R. y Rosales J. (2013). Psicoanálisis en la actualidad, en Salud Mental o Mental Health, Buenos Aires: Ed. Serie Conexiones.

Dennett, D. (2014) Internet se vendrá abajo y viviremos oleadas de pánico, disponible en: http://cultura.elpais.com/cultura/2014/03/25/act ualidad/1395776953_258137.html?id_externo_ rsoc=FB_CM

De Kunder, M. (2017). The size of the World Wide Web (The Internet). Consultado en: http://www.worldwidewebsize.com/

Descifremos, Cifras de la Organización Mundial de la Salud, (2016) Disponible en https://www.gob.mx/cms/uploads/attachment/fi le/280081/descifremos15-2.pdf

Consulado 2 de septiembre de 2019.

Dewey, C. (2015). If you could print out the whole Internet, how many pages would it be? Washington Post, 18.

Facebook.com

Familiar, F. "Qué tal Fernanda" programa de Imagen Radio, marzo 2018

Freud, S. (1915/1986) Conferencias de introducción al psicoanálisis, " $1^{a}$ conferencia. Introducción”, En J. Strachey (Ed.), Sigmund Freud obras completas. (J.L. Etcheverry, Trad. Vol. XV., p. 15). Buenos Aires: Amorrortu. 
Freud, S. (1930 [1929]/2017). El malestar en la cultura En J. Strachey (Ed.), Sigmund Freud obras completas. (J.L. Etcheverry, Trad. Vol. XXI) Buenos Aires: Amorrortu.

Freud, S. (1926/2017). ¿Pueden los legos ejercer el psicoanálisis?, En J. Strachey (Ed.), Sigmund Freud obras completas. (J.L. Etcheverry, Trad. Vol. XX., p. 80). Buenos Aires: Amorrortu.

García Sánchez, C. (2019). El inframundo de internet. Canal Triline, YouTube http://bit.ly/POqbZu

Gorenstein, A. (2012) "Crece la terapia online: la usan uno de cada tres psicólogos" en http://www.clarin.com/tendencias/Villa-FreudWeb_0_750524996.html, 05.08.2012, consultada 30.06.2016

Google. (2017). Informe de transparencia: Solicitudes gubernamentales de retirada de contenido. Consultado en: https://www.google.com/transparencyreport/re movals/government/

Hamilton, S. (2014). Conferencia: Engaging and Supporting Today's Learners. Directora Ejecutiva de Apple Education. Cupertino, Califormia USA. Impartida en Universidad Autónoma de Querétaro, (México). 25 de marzo de 2014

Hilbert. M. (2017). Martin Hilbert, experto en redes digitales: "Obama y Trump usaron el big data para lavar cerebros", disponible en: http://www.theclinic.cl/2017/01/19/martinhilbert-experto-redes-digitales-obama-trumpusaron-big-data-lavar-cerebros/, consultado el 25 de enero de 2017

Jalife-Rahme, A. (2017) Bajo la Lupa, en: http://www.jornada.unam.mx/2016/11/23/opini on/018o1 pol Consultado el 2 de febrero de 2017

Lacan, J. (1952). El mito individual del neurótico. Versión inédita.

Lacan, J. (1960/1995). Seminario 7 "La Ética del Psicoanálisis". 10.02.60. Buenos Aires: Paidós. (D. Ravinovich, Trad.) p. 171.
Lacan, J. (1966) Psicoanálisis y Medicina. El lugar del psicoanálisis en medicina, Conferencia Collegede Médecine, en La Salpetrtiere, 16 de febrero de 1966, texto inédito

Lacan, J. (1975). Conférences dans les universités nord-américaines: le 2 décembre 1975 au Massachusetts Institute of Technology. Scilicet(6-7), 53-63.

Lacan, J. (1969). Seminario 16 "De un Otro al otro". 12.03.69. Buenos Aires: Escuela Freudiana de Buenos Aires. (Versión inédita) (A. M. Gómez y S. Rocchietti, Trad.)

Lacan, J. (1973/1981). Seminario 20 "Aún". 13.03.73. Buenos Aires: Paidós. (D. Ravinovich, Delmont-Mauri y J. Sucre, Trad.) p. 99.

Lacan, J. (1974/1980). "La Tercera, 01.11.74". Actas de la Escuela Freudiana de París. Barcelona: Ed. Petrel., p. 186.

Lacan, J. (1970-74/1993). Psicoanálisis, Radiofonía y Televisión, (O. Massota y O. Gimeno-Grendi Trad). Barcelona: Ed. Anagrama.

Lacan, J. (1974/2005). El triunfo de la religión. (N. González Trad.) Buenos Aires: Paidós.

Leigh D. y Harding L. (2011) Wikileaks y Assange, (M. Vidal e I. Merino Trad) Barcelona: Ed. Deusto.

Lerman R. y M. Gordon. (2019). Google tendrá que pagar 170 millones de dólares por violar la privacidad de los niños en YouTube. Associate Press, disponible en https://espanol.yahoo.com/finanzas/noticias/mu ltan-youtube-eeuu-170-millones-

131935993.html

4 de septiembre de 2019, consultado 4 de septiembre de 2019

Lipovetsky, G. (1990) El imperio de lo efímero, Barcelona: Anagrama.

López C. "IA: ¿qué falta”. (enero 2018). Blog Psicoanálisis México, en http://www.psicoanalisismexico.com/reflexiones/artenero18A.html, consultado 22 de mayo 2018 
Meserve, S. A., \& Pemstein, D. (2017). Google Politics: The Political Determinants of Internet Censorship in Democracies. Political Science Research and Methods, 1-19

Molina, A. (2016) El servicio de gmail supera los mil milones de usuarios activos al mes, en HTTPS://MARKETING4ECOMMER CE.NET/CORREO-GMAIL-1000-

MILLONES-USUARIOS /, consultado 23 de mayo de 2018

Pappas, S. (2016). How big is the internet, really. Live Science, 18.

Peirone, F. (2016) El psicoanálisis en la era de la hiperconectividad, disponible en: https://www.pagina12.com.ar/diario/psicologia/ 9-312734-2016-10-27.html, consultado 30 octubre 2016.

Periódico 24 horas. (2019). El Diario sin límites, México tiene 125 millones de habitantes: INEGI, Granados Alejandro, 8 de mayo de 2019, en https://www.24-horas.mx/2019/05/08/mexicotiene-125-millones-de-habitantes-inegi/, consultado el 2 de septiembre de 2019

Periódico El País. (2019). Se compran amigos y abrazos: la epidemia de soledad en EEUU ya es un negocio, Antonia Laborde, en https://elpais.com/sociedad/2019/07/29/actualid ad/1564417043_013460.html, 26 de agosto de 2019, consultado 30 de agosto de 2019

Periódico El País. (2018) Zuckerberg rinde cuentas ante la Eurocámara por escándalo de datos personales, 22 mayo 2018, en: https://www.elpais.com.uy/vida-

actual/zuckerberg-rinde-cuentas-eurocamaraescandalo-datos-personales.html, consultado 25 de mayo 2018

Periódico Infobae. (2019). Apple admitió que escuchó conversaciones de Siri sin autorización y ofreció una disculpa. Disponible en: https://www.infobae.com/america/tecno/2019/0 8/28/apple-admitio-que-escuchoconversaciones-de-siri-sin-autorizacion-yofrecio-una-disculpa/, 28 de agosto de 2019, consultado 3 de septiembre de 2019
Periódico Sin Embargo, Alumnos del IPN crean un software que ayuda a estimular la memoria de pacientes con alzheimer, Riquelme Rodrigo, 12 de julio de 2019 , consultado 30 de agosto de 2019, en https://www.sinembargo.mx/12-072019/3612131

Recalcati, M. (2008) Clínica del vacío. Anorexias, dependencias, psicosis. (S. Rodríguez Trad.) Madrid: Síntesis

Rodriguez, S. (2016). Psicoanálisis online, http://www.pagina12.com.ar/diario/psicologia/9 -253406-2014-08- 21.html

21.08.2014, Consultado 12.09.2016

Sabbadini, A. (2013) New technologies and the psychoanalytic setting, en MyiLybrari, London.

Soria, M. (mayo 2018). El psicoanálisis y un mundo híper-conectado. Universidad Nacional de Córdoba, Argentina, en: HTTPS://ELRESORTE.NET/2018/05/08/ELPSICOANALISIS-Y-UN-MUNDO-HIPERCONECTADO/, Consultado 26 mayo 2018.

Telemundo Noticias, Facebook crea una aplicación de alerta de tiroteos y fenómenos naturales, 28 de agosto de 2019, en https://www.telemundo.com/noticias

Tisseron, S. (2011) La intimidad sobreexpuesta, París: Ramsay, 2001

Trujillo, H. (2016) Testimonio de un paciente de psicoanálisis online http://www.htpsicoanalisis.com/testimonio-deun-paciente-de-psicoanalisis-online/

Consultado 06.06.2016

Twitter.com

Valery, Y. (2016). Cómo averiguar todo lo que Google sabe de ti [Press release]. Consultado en: http://www.bbc.com/mundo/noticias-36797839

\section{YouTube.com}

Zizec, S. (2006/2013). "Dios está muerto, pero no lo sabe". Lacan juega con Bobok en Como leer a Lacan. Buenos Aires: Paidós 